\title{
Abnormal Ocular Findings in Chronic Renal Failure Patients on Hemodialysis
}

\author{
Dina E. Mansour, Ahmed M. El-Bayomi, Aml M. El-Azab * \\ Department of Ophthalmology, Faculty of Medicine, Ain-Shams University \\ * Corresponding author: Aml M. El-Azab; Mobile: 01019635327. Email: amlnoor87@yahoo.com
}

\begin{abstract}
Background: chronic kidney disease (CKD) is a global public health problem and diabetes is a leading cause for it. Those patients with end-stage renal disease (ESRD) are generally treated using hemodialysis (HD). HD causes numerous metabolic changes which in turn induce osmotic changes in blood and extracellular fluids including the aqueous and vitreous humor, so there are many ocular abnormalities in ESRD patients either due to common pathophysiological mechanisms and risk factors between eye and kidney diseases or due to hemodialysis itself. Aim of the Work: the aim of this study is to evaluate ocular findings in CKD patients on hemodialysis, including the best-corrected visual acuity (BCVA), measurement of intraocular pressure (IOP), corneal, conjunctival, tear film changes, lens and fundus changes. Patients and Methods: this cross sectional observational study was carried out on twenty three patients (6 males and 17 females) with 44 eyes, with chronic kidney disease on chronic hemodialysis for 2-10 years in the Dialysis Unit at Agouza Specialized Hospital in the period from November 2017 to April 2018. The hemodialysis duration was approximately four hours three times per week. Results: forty three percent of total 44 eyes had BCVA > 6/18. As regard anterior segment, we found yellow sclera was the commonest finding (75\%), cataract (61.4\%), lid edema (56.8\%), dry eye (43.2\%), conjunctival congestion (47.7\%), and pterygium (13.6\%). As regard posterior segment; Diabetic retinopathy was the commonest finding (38.6\%), hypertensive retinopathy (4.5\%), AMD (2.3\%), macular hemorrhage $(2.3 \%)$. Regarding OCT findings, there was statistically significant difference between eyes of diabetic, hypertensive and other patients as regard central foveal thickness. Conclusion: our results demonstrate high prevalence of abnormal ocular findings in chronic renal failure patients on hemodialysis. So those patients should be evaluated regularly by the ophthalmologist.
\end{abstract}

Keywords: Chronic Renal Failure, Hemodialysis, Ocular Findings, Central Foveal Thickness, OCT.

\section{INTRODUCTION}

Chronic kidney disease (CKD) is a slow progressive loss of kidney function over a period of several years, which also is the presence of either reduced renal function as evaluated by estimated glomerular filtration rate (eGFR $<60$ $\mathrm{ml} /$ minute $/ 1.73 \mathrm{~m}^{2}$ ) or albuminuria evaluated by urinary albumin to creatinine ratio (ACR $\geq 30$ $\mathrm{mg} / \mathrm{g})^{(1)}$. Chronic kidney disease has become a major public health problem worldwide and has been associated with premature morbidity and mortality ${ }^{(2)}$. Chronic kidney disease has numerous causes. The most common causes of CKD are diabetes mellitus and long-term uncontrolled hypertension ${ }^{(3)}$. Chronic renal failure patients have had a wide range of findings, including refractive changes, dry eye, increased tear osmolarity, conjunctival calcium deposits, band keratopathy, corneal endothelium changes and lenticular opacity ${ }^{(4)}$. Patients with chronic kidney disease are generally treated using a blood filtration mechanism such as hemodialysis. The primary objective of hemodialysis is to correct the composition and volume of body fluids. Correction of body fluid aims to resolve the excessive accumulation and abnormal distribution of body fluids and is concerned with the change in plasma colloid osmotic pressure during haemodialysis ${ }^{(5)}$. During hemodialysis, numerous metabolic parameters including blood urea, sodium, potassium and sugar will be changed ${ }^{(\boldsymbol{)}}$, which also induce osmotic changes in blood and extracellular fluids. This includes the aqueous and vitreous humor, any change in osmotic pressure of these fluids could affect the refractive status ${ }^{(7)}$. The relationship between chronic hemodialysis and intra-ocular pressure (IOP) has been widely investigated in the past. There are several studies, which demonstrate a significantly increased IOP and decreased OPP occur during $\mathrm{HD}$, bringing both to levels that increase the risk of glaucoma development and progression ${ }^{(8)}$. In contrast, other studies have demonstrated the opposite effect ${ }^{(9)}$, while some studies reported no IOP change during HD ${ }^{(10)}$. Ocular posterior segment changes such as, retinopathy secondary to diabetes mellitus, hypertension, anemia, and uremia, are also observed frequently in CRF patients ${ }^{(11)}$. Pahor et al. found that central foveal thickness was significantly thinner in hemodialysis patients than normal healthy subjects ${ }^{(\mathbf{1 2})}$.

\section{AIM OF THE WORK}

The aim of this study is to evaluate ocular findings in CKD patients on hemodialysis, including 
the best-corrected visual acuity (BCVA), measurement of intraocular pressure (IOP), corneal, conjunctival, tear film changes, lens and fundus changes.

\section{PATIENTS AND METHODS}

Subjects: This cross sectional observational study was carried out on twenty three patients (6 males and 17 females) with 44 eyes, with chronic kidney disease on chronic hemodialysis for 2-10 years in the Dialysis Unit at Agouza Specialized Hospital in the period from November 2017 to April 2017. The hemodialysis duration was approximately four hours three times per week. The study was approved by the Ethics Board of Ain Shams University and an informed written consent was taken from each participant in the study.

Inclusion criteria: 1. The patients can cooperate with full ophthalmological examination. 2. All our patients previously diagnosed with chronic kidney disease were above 18 years of age. 3 . Each patient received an average of 3-4 hours hemodialysis sessions, 2-3 times per week for 2-10 years using a high-performance dialyzer at a blood flow rate of $250 \mathrm{ml} / \mathrm{min}$.

Exclusion criteria: 1 . The presence of any ocular disease that would interfere with retinal examination and/or IOP measurement: corneal opacity and dense cataract. 2. History of ocular trauma within 3 months.

Sampling: Patients who were convenient to our study were enrolled after consideration of inclusion and exclusion criteria and obtaining an informed verbal consent from all of them.

Methods: All our cases were subjected to full ophthalmological examination after completing their hemodialysis session included: 1. Thorough medical history taking, including age, sex, cause of $\mathrm{CRF}$, duration of dialysis, presence and duration of diabetes mellitus, presence and duration of hypertension (HTN) and drug history. 2. Past ophthalmic history was taken, including history of glasses, ocular medication, and ocular surgeries. 3. Assessment of visual acuity using a Snellen chart for distant vision, Best corrected visual acuity using an auto - refractometer. 4. Measurement of IOP by Goldmann applanation tonometry. 5 . Examination of EOM in all gazes. 6. Examination of Anterior segment by Slit lamp Biomicroscopy including eyelids, lid lashes, cornea, conjunctiva, measurement of tear film BUT after staining with fluorescent, pupil (diameter, shape, reaction to light), anterior chamber for cells or flare, and depth. Inspection of iris for color, pattern, and abnormal vessels. Lens examination was done. 7. Fundus examination using Slit lamp Biomicroscopy with +90 Diopter lens after pupil dilatation with tropicamide. This included examination of macula, optic disc, retinal vessels and background. 8. Fundus photographs were taken by fundus camera built in OCT device. 9. Macular assessment regarding thickness and presence of maculopathy was done after hemodialysis session using Topcon SD-OCT-2000 ver.8.30 (512x128). A 3D Macular scanning protocol is selected and a live OCT window was seen. The results were analyzed and macular mapping was obtained using Optovue RTVue software to obtain a macular thickness map. Statistical analysis: The collected information was recorded, coded, tabulated and analyzed for statistical purposes by utilizing the Statistical Package for Social Science (SPSS) version 22 for Windows on personal computers. Qualitative information was described as percentage and numbers. Quantitative information was described as means $[ \pm$ standard deviation (SD)] for parametric variable or medians (minimum - maximum), for non-parametric variables, as suitable. Testing for normality was done by using Kolmogorov-Smirnov test. And, for comparing between groups; t-test was used for normally distributed variables; while Chi-square test was used for qualitative variables. $\mathrm{P}$ value $\leq 0.05$ was considered to be statistically significant.

\section{RESULTS}

In our study a total of 44 eyes of 23 chronic kidney disease patients on hemodialysis (6 males and 17 females) were enrolled. Frequency of hemodialysis per week was 3 times. Their age ranged from 31 to 60 years (The mean age 50.95 with SD 8.15 years). Twenty three of eyes were right and twenty one were left; table 1 .

Table (1): Demographic data of study patients $(\mathrm{n}=23)$

\begin{tabular}{|l|c|}
\hline Gender Male (n, \%) & $6,26.1 \%$ \\
Female (n, \%) & $17,73.9 \%$ \\
\hline Age (years; Mean \pm SD) & $50.95 \pm 8.15$ \\
(Min-Max) & $31-60$ \\
\hline Right eye (n, \%) & $23,52.3 \%$ \\
Left eye (n, \%) & $21,47.7 \%$ \\
\hline
\end{tabular}


Regarding the medical history of patients, 9 patients with percent of $39.1 \%$ were diabetic while 7 patients with percent of $30.4 \%$ were hypertensive and 2 patients $(8.7 \%)$ had bronchial asthma. The mean duration of hemodialysis was 6.60 years with SD 2.06. Past ophthalmic history showed that 6 of patients with percent of $26.1 \%$ had history of wearing glasses. Medical and past ophthalmic histories of the patients are shown in table 2 .

Table (2): Medical history and past ophthalmic history of studied patients $(\mathrm{n}=23)$

\begin{tabular}{|l|c|}
\hline DM (n, \%) & $9,39.1 \%$ \\
\hline HTN (n, \%) & $7,30.4 \%$ \\
\hline BA (n, \%) & $2,8.7 \%$ \\
\hline $\begin{array}{l}\text { Duration of hemodialysis in years } \\
\text { (mean } \pm \text { SD) }\end{array}$ & $6.60 \pm 2.06$ \\
\hline Past ophthalmic history: glasses (n, \%) & $6,26.1 \%$ \\
\hline
\end{tabular}

In our study, causes of CKD were DM 9 patients out of total 23 (39.1\%) which represents the most prevalent cause in our study, HTN 7 (30.4\%), both DM and HTN were recorded in one patient. Three patients (13\%) had CKD due to chronic glomerulonephritis. Analgesic nephropathy was the cause in 2 patients $(8.7 \%)$ and one patient had lupus nephritis by percent of $4.3 \%$. Two patients $(8.7 \%$ ) had CKD due to unknown cause. Ocular findings in CRF patients on HD included in our study: according to WHO criteria, best corrected visual acuity in the forty four eyes ranged from good vision $(>6 / 18)$ to legally blind $(<6 / 60)$, in our study 16 eyes $(36.4 \%)$ reported BCVA of $6 / 6$, nine eyes $(20.5 \%$ ) with BCVA $>6 / 18$. Twelve eyes $(27.3 \%)$ were recorded with impaired vision ranging from $6 / 24$ to $6 / 60$. The records showed that 7 eyes were legally blind, six of them $(13.6 \%$ of total eyes) with BCVA less than $6 / 60$ and one eye was seeing hand movement; $2.3 \%$ (Table 3).

Table (3): BCVA in the eyes of studied groups $(\mathrm{n}=44)$

\begin{tabular}{|l|c|c|c|}
\hline WHO criteria & Visual acuity & Number & Percentage \\
\hline \multirow{2}{*}{ Good vision } & $6 / 6$ & 16 & $36.4 \%$ \\
& $>6 / 18$ & 9 & $20.5 \%$ \\
\hline Impaired vision & $6 / 24-6 / 60$ & 12 & $27.3 \%$ \\
\hline \multirow{2}{*}{ Legally blind } & $<6 / 60$ & 6 & $13.6 \%$ \\
& $\mathrm{HM}$ & 1 & $2.3 \%$ \\
\hline Total eyes & & 44 & $100 \%$ \\
\hline
\end{tabular}

Nineteen eyes (43.2\%) had BCVA of less than 6/18, major causes were cataract in 9 eyes (20.4\%) of total 44 eyes and DME in 7 eyes $(15.9 \%)$ followed by PDR in two eyes (4.5\%). Age related macular degeneration was the cause in one eye $(2.3 \%)$. Causes of impaired visual acuity less than $6 / 18$ are shown in table (4);

Table (4): Causes of visual impairment in the eyes with BCVA $<6 / 18$

\begin{tabular}{|l|c|c|}
\hline \multicolumn{1}{|c|}{ Cause } & Number & Percentage \\
\hline Cataract & 9 & $20.4 \%$ \\
\hline DME & 7 & $15.9 \%$ \\
\hline PDR & 2 & $4.5 \% \%$ \\
\hline AMD & 1 & $2.3 \%$ \\
\hline Total eyes & 19 & $43.2 \%$ \\
\hline
\end{tabular}

Table (5) shows IOP measurement after HD session, the mean IOP was 12.68 with SD $1.92 \mathrm{~mm}$ $\mathrm{Hg}$ ranging from 10 to $16 \mathrm{~mm} \mathrm{Hg}$, there was statistically significant difference among those who had DM, HTN and neither as regard the mean IOP (P-value < 0.001); in diabetic patients the mean IOP was 12.4 with SD $1.72 \mathrm{~mm} \mathrm{Hg}$ and in hypertensive the mean IOP was 14.72 with SD $1.42 \mathrm{~mm} \mathrm{Hg}$. Others (non-diabetic, non-hypertensive) had a mean of IOP equals 11.66 with SD $1.41 \mathrm{~mm} \mathrm{Hg}$.

Table (5): IOP after hemodialysis session

\begin{tabular}{|c|c|c|c|c|}
\hline & \multicolumn{2}{|c|}{ Mean \pm SD } & \multicolumn{2}{c|}{ Min-Max } \\
\hline $\begin{array}{l}\text { IOP } \\
(\mathrm{mm}\end{array}$ & \multicolumn{2}{|c|}{$12.68 \pm 1.92$} & \multicolumn{2}{|c|}{$10-16$} \\
$\mathrm{Hg})$ & \multicolumn{2}{|c|}{} & \multicolumn{2}{|c|}{} \\
\hline & DM & HTN & Others & P-value \\
\hline & $12.4 \pm 1.72$ & $14.72 \pm 1.42$ & $11.66 \pm 1.41$ & $<0.001$ \\
\hline
\end{tabular}

Abnormal anterior segment ocular findings as shown in table (6), yellow sclera was the commonest finding in 33 eyes $(75 \%)$, there was no statistically significant difference between diabetic, hypertensive and others as regard yellow sclera (Pvalue $=0.939$ ). The second common finding was cataract in 27 eyes $(61.4 \%)$, there was no statistically significant difference between diabetic, hypertensive and others as regard cataract. Lid edema was in 25 eyes (56.8\%) with no statistically significant difference between diabetic, hypertensive and others as regard lid edema ( $\mathrm{P}$ value $=0.245$ ). Dry eye was found in 19 eyes (43.2\%) with no statistically significant difference between diabetic, hypertensive and others (Pvalue $=0.505)$. Regarding conjunctiva, there was conjunctival congestion in 21 eyes (47.7\%). Pterygium was found in six eyes of hypertensive patients (13.6\%), two eyes had PTDs (4.5\%), there was statistically significant difference between diabetic, hypertensive and others as regard conjunctival congestion, pterygium and PTDs (P- 
value $=0.003$ ). The nebula was found in one eye (2.3\%). Two eyes had IOL (4.5\%).

Table (6): Abnormal anterior segment findings as regard 1ry diseases causing chronic kidney disease

\begin{tabular}{|l|c|c|c|c|c|c|}
\hline & DM & HTN & Others & Total & Percentage & P value \\
\hline Lid edema & 11 & 6 & 8 & 25 & $56.8 \%$ & 0.245 \\
\hline Dry eye & 7 & 6 & 6 & 19 & $43.2 \%$ & 0.505 \\
\hline Conjunctiva & & & & & & \\
congestion & 8 & 3 & 10 & 21 & $47.7 \%$ & 0.003 \\
Pterygium & 0 & 6 & 0 & 6 & $13.6 \%$ & \\
PTDs & 0 & 0 & 2 & 2 & $4.5 \%$ & \\
\hline Yellow sclera & 11 & 8 & 14 & 33 & $75 \%$ & 0.939 \\
\hline Nebula & 0 & 1 & 0 & 1 & $2.3 \%$ & 0.215 \\
\hline Cataract & 11 & 8 & 8 & 27 & $61.4 \%$ & 0.261 \\
\hline
\end{tabular}

The most important and vision threatening were the posterior segment findings found in 21 eyes $(47.7 \%)$ as shown in table (7). Diabetic retinopathy was the commonest finding presented in nonproliferative DR without clinically significant macular edema in 8 eyes (18.2\%), non-proliferative DR with CSME in 7 eyes (15.9\%) and proliferative DR with CSME in two eyes (4.5\%). Two eyes had hypertensive retinopathy $(4.5 \%)$. One eye had age related macular degeneration $(2.3 \%)$. Macular hemorrhage was found in one eye of hypertensive patient (2.3\%).

Table (7): Abnormal posterior segment findings in studied group $(\mathrm{n}=44)$

\begin{tabular}{|l|c|c|}
\hline & Number & Percentage \\
\hline NPDR only & 8 & $18.2 \%$ \\
NPDR \& CSME & 7 & $15.9 \%$ \\
PDR \& CSME & 2 & $4.5 \%$ \\
\hline Hypertensive retinopathy & 2 & $4.5 \%$ \\
\hline Macular Hemorrhage & 1 & $2.3 \%$ \\
\hline Degenerative & 1 & $2.3 \%$ \\
\hline Normal & 23 & $52.3 \%$ \\
\hline
\end{tabular}

OCT was used in our study for macular assessment and OCT findings were found in 13 eyes (29.5\%) and recorded as shown in table (8), diabetic macular edema (DME) was the commonest finding as it was present in 11 eyes out of total 44 eyes (25\%); Age related macular degeneration and macular hemorrhage were equally present each in one eye by (2.3\%).

Table (8): Abnormal OCT finding in studied group $(\mathrm{n}=44)$

\begin{tabular}{|l|c|c|}
\hline & Number & Percentage \\
\hline DME & 11 & $25 \%$ \\
\hline AMD & 1 & $2.3 \%$ \\
\hline Macular hemorrhage & 1 & $2.3 \%$ \\
\hline Total & 13 & $29.5 \%$ \\
\hline
\end{tabular}

OCT had given us an idea about macular thickness through macular mapping, there was statistically significant difference between eyes of diabetic, hypertensive and other patients as regard central foveal thickness (CFT); (P-value $=0.046)$. Mean CFT in eyes of diabetic patients was the highest, as it was $319.26 \mu \mathrm{m}$ with SD $139.4 \mu \mathrm{m}$, whereas mean CFT in hypertensive patients was $264 \mu \mathrm{m}$ with SD $28.2 \mu \mathrm{m}$. In non-diabetic nonhypertensive eyes mean CFT was $234.05 \mu \mathrm{m}$ with SD $73.14 \mu \mathrm{m}$; table $(\boldsymbol{9})$.

Table (9): Comparison between CFT of patients as regard 1ry cause of CKD

\begin{tabular}{|l|c|c|c|c|}
\hline & DM & HTN & Others & $\begin{array}{c}\text { P } \\
\text { value }\end{array}$ \\
\hline $\begin{array}{l}\text { CFT }, \mu \mathrm{m} \\
(\text { mean } \pm \text { SD })\end{array}$ & $319.26 \pm 139.4$ & $264 \pm 28.2$ & $234.05 \pm 73.14$ & 0.046 \\
\hline
\end{tabular}

\section{DISCUSSION}

In our study, ESRD was more frequent in female patients than males which agreed with the most recent US Renal Data System (USRDS) annual data report; the prevalence of chronic renal failure between the years 2007 and 2012 was higher in women $(15.1 \%)$ than in men $(12.1 \%)^{(13)}$. The age of our patients ranged from 31 to 60 years (The mean age 50.95 with SD 8.15 years). Renal function decreases with age in both men and women. Thus, the elderly population is more prone to develop CKD after various renal insults ${ }^{(14)}$. In our work, DM and HTN were the main causes of chronic kidney disease; this agreed with Hernandez and Nasri ${ }^{(15)}$. As regards ocular findings that we found in our study, BCVA represented by $57 \%$ of patients had good vision more than $6 / 6$, this result was close to many studies ${ }^{(16-17)}$. However, visual acuity is not the only indicator of the ocular status. Even advanced DR and HR, may have good central vision until macula is involved ${ }^{(16-17)}$.In our study 10 out of 17 eyes with diabetic retinopathy and two eyes with hypertensive retinopathy had quiet good vision but were at risk of blindness. Dahal and Gouli stated that hemodialysis patients, especially the elderly, have visual acuity (VA) levels much lower than their age-matched counterparts (16). There was no control group in our work. In our study, $43 \%$ of eyes in our study had BCVA of less than 6/18. Cataract was the main cause of impaired vision less than $6 / 18$ which was seen in cases by $20 \%$ that agree with Dahal and Gouli ${ }^{(16)}$, but in a 
similar study by Malleswari et al. hypertensive retinopathy was the cause of visual loss in the majority, but cataract was in a significant number represented by $38 \%$ of examined eyes ${ }^{(17)}$. Diabetic maculopathy and DR caused visual impairment of eyes in $20.5 \%$ of cases. An earlier study by Bhajracharya et al., recorded that the main cause of impaired vision less than $6 / 18$ was maculopathy (18). In our study, age related macular degeneration was recorded in one eye, this agreed with Mathew et al. who found AMD by $2.5 \%{ }^{(\mathbf{1 9})}$. Mean intra ocular pressure in patients of CRF in our study was within normal range $12.68 \pm 1.92 \mathrm{~mm} \mathrm{Hg}$. There were similar studies found the same result ${ }^{(16-19)}$. Lid edema in hemodialysis patients in our work was found in 25 eyes (56.8\%), which agree with many studies ${ }^{(\mathbf{1 6}-18)}$. Dry eye was found in 19 eyes $(43.2 \%)$ with no statistically significant difference between diabetic, hypertensive and others. A similar study done by Jung et al. in 2013, they found that dry eye was consistent and significant before and after HD and cornea turned dry with no statistically significant difference between DM and non-diabetic patients ${ }^{(5)}$. Regarding conjunctiva, there was conjunctival congestion in 21 eyes (47.7\%), which agree with Dahal and Gouli who reported congestion in 150 of their cases $(50 \%)^{(16)}$. Pterygium was found in six eyes of hypertensive patients $(13.6 \%)$. Cataract was found in 27 eyes $(61 \%)$ and this resembled a study done by Mathew et al., percent of cataract was $64 \%{ }^{(19)}$ and Bhajracharya et al., had found that $18 \%$ of CKD patients had cataract ${ }^{(18)}$. In a study done by Malleswari et al., prevalence of cataract was $38 \%$ (17). The present study showed a high prevalence which may be due to the fact that patients presented at a later stage in the course of CKD. Cataract in ESRD patients can be explained by comorbidities, these include advanced age, diabetes, hypertension, the use of corticosteroids, ultraviolet light exposure and hyperparathyroidism (20). In our work, posterior segment findings were the most visual threatening signs which were found in 21 eyes $(47.7 \%)$ of which diabetic retinopathy was the commonest finding 17 eyes $(38.5 \%)$ of which 15 eyes out of total 44 eyes (34\%) had mild to moderate NPDR and two eyes (4.5\%) had PDR. Also clinically significant macular edema was found in 9 eyes $(20.8 \%)$ related to diabetic retinopathy and one eye related to both DR and HR. Findings correlated well with other studies and diabetic retinopathy was consistent and significant finding in ESRD patients, as for example, Malleswari et al. recorded that in total $31 \%$ of diabetic retinopathy patients, NPDR was seen in $20 \%$, PDR in $6 \%$, maculopathy in $5 \%$ of the cases ${ }^{(17)}$. In our study we found two eyes had hypertensive retinopathy and this agreed with many studies in which HR was significant and consistent finding ${ }^{(\mathbf{1 6}-19)}$. Only one eye was manifested by macular hemorrhage in hypertensive patient. Deva et al. reported similar finding ${ }^{(21)}$. In our work, there was one eye having age related macular degeneration. This agreed with a prospective cohort study by Liew et al. who suggested that patients with chronic kidney disease are at much greater risk of developing AMD compared to the general population ${ }^{(22)}$. Also, many studies reported AMD with similar results in ESRD patients (19-23). In our study, there was no retinal detachment as reported by Dahal and Gouli (16). Neither BRVO nor glaucomatous optic neuropathy found by Malleswari et al. was seen in our study ${ }^{(17)}$. OCT was used for macular assessment and OCT findings were found in 13 eyes (29.6\%) and recorded, diabetic macular edema (DME) was the commonest finding as it was present in 11 eyes out of total 44 eyes (25\%). Age related macular degeneration and macular hemorrhage were equally present each in one eye (2.3\%). Our results correlated to clinical findings by ophthalmoscopy. In our work, there was statistically significant difference between eyes of diabetic, hypertensive and other patients as regard CFT (P-value=0.046). Mean CFT in eyes of diabetic patients was the highest, as after dialysis session, it was $319.26 \mu \mathrm{m}$ with SD $139.4 \mu \mathrm{m}$, whereas mean CFT in hypertensive patients was $264 \mu \mathrm{m}$ with SD $28.2 \mu \mathrm{m}$. In non-diabetic non-hypertensive eyes mean CFT was $234.05 \mu \mathrm{m}$ with SD $73.14 \mu \mathrm{m}$. In a similar study by Chelala et al. after dialysis session, the mean CFT in diabetic patients was $283.50 \pm 57.12$ and that of hypertensive patients was $252.80 \pm 29.62$ and patients with other causes had a mean CFT of 240.42 $\pm 26.31^{(24)}$. High thickness in DM is due to presence of macular edema.

\section{CONCLUSION}

Our results demonstrate high prevalence of abnormal ocular findings in chronic renal failure patients on hemodialysis as regard vision, anterior and posterior segments abnormalities which need close follow up to those patients on chronic hemodialysis by the ophthalmologist. 


\section{CONFLICTS OF INTEREST}

There are no conflicts of interest.

\section{REFERENCES}

1- Jiang L, Liang Y, Qiu B et al. (2010): Prevalence of chronic kidney disease in a rural Chinese adult population: the Handan Eye Study. Nephron Clin Pract., 114(4):c295-302.

2- Coresh J, Selvin E, Stevens LA et al. (2007): Prevalence of Chronic Kidney Disease in the United States. JAMA., 298(17):2038-2047.

3- Kes P, Basić-Jukić R, Ljutić D, BrunettaGavranić B. (2011): The role of arterial hypertension in the development of chronic renal failure. Acta Medica Croatica (in Croatian), 65(3):78-84.

4- Diaz-Couchoud P, Bordas FD, Garcia JR et al. (2001): Corneal disease in patients with chronic renal insufficiency undergoing hemodialysis. Cornea, 20:695-702.

5- Jung JW, Yoon MH, Lee SW, Chin HS (2013): Effect of hemodialysis (HD) on intraocular pressure, ocular surface, and macular change in patients with chronic renal failure. Effect of hemodialysis on the ophthalmologic findings. Graefes Arch Clin Exp Ophthalmol., 251:153162.

6- Gotch FA, Panlilio FM, Buyaki RA et al. (2004): Mechanisms determining the ratio of conductivity clearance to urea clearance. Kidney Int Suppl., (89):S3-24.

7- Lin SF, Lin PK, Chang FL, Tsai RK (2009) Transient hyperopia after intensive treatment of hyperglycemia in newly diagnosed diabetes. Ophthalmologica, 223:68-71.

8- Hu J, Bui KM, Patel KH et al. (2013): Effect of Hemodialysis on Intraocular Pressure and Ocular Perfusion Pressure. JAMA Ophthalmol., 131(12):1525-31.

9- Afshar R, Ghasemi H, Shabpiray $H$ et al. (2013): Monitoring of intraocular pressure and its correlation with systemic parameters before and after hemodialysis. Iran J Kidney Dis., 7(1):53-9.
10- Samsudin A, Mimiwatti Z, Soong $T$ et al. (2010): Effect of hemodialysis on intraocular pressure. Eye, 24:70-3.

11- Hamed LM, Winward KE, Glaser JS, Schatz NJ (1989): Optic neuropathy in uremia. Am J Ophthalmol., 108(1):30-35.

12- Pahor D, Gracner B, Gracner T, Hojs $R$ (2008): Optical coherence tomography findings in hemodialysis patients. Klin Monbl Augenheilkd, 225:713-717.

13- Collins AJ, Foley RN, Herzog C et al. (2011): US Renal Data System 2010 Annual Data Report. American Journal of Kidney Diseases, 57(1):e1-526.

14- Falodia J, Singla MK (2012): CKD epidemiology and risk factors. Clin Queries Nephrol., 1:249-252.

15- Hernandez GT, Nasri H (2014): World Kidney Day 2014: increasing awareness of chronic kidney disease and aging. $\mathbf{J}$ Renal Inj Prev., 3(1):3-4.

16- Dahal P and Gouli S (2014): Ocular findings in the chronic renal failure. Journal of College of Medical SciencesNepal, 10:18-24.

17- Malleswari B, Rahmathunnisa, Irshad (2016): Eye findings in chronic renal failure patients undergoing hemodialysis. International Journal of Contemporary Medical Research, 3(7): 1912-1914.

18- Bhajracharya L, Shah DN, Raut KB, Koirala S (2008): Ocular evaluation in patients with Chronic Renal Failure-A hospital based Study. Nepal Med Coll J., 10(4):209-14.

19- Mathew RG, Abraham G, Prasanth HR (2016): Ocular Changes in Chronic Kidney Disease. Journal of Ophthalmology \& Visual Neurosciences, 5(2):1-5.

20- Evans RD, Rosner M (2005): Ocular abnormalities associated with advanced kidney disease and hemodialysis. Semin Dial., 18 (3): 252-257. 
Dina E. Mansour et al.

21- Deva R, Alias MA, Colville D et al. (2011): Vision-threatening retinal abnormalities in chronic kidney disease stages 3 to 5 . Clinical Journal of the American Society of Nephrology, 6(8):1866-71.

22- Liew G, Mitchell P, Wong TY et al. (2008): CKD increases the risk of agerelated macular degeneration. J Am Soc Nephrol., 19:806-811.
23- Joju G, Kakkanatt CVA, Mathai M et al. (2017): Ocular manifestations in diabetes-related chronic renal failure patients in a tertiary care centre. J. Evid. Based Med. Healthc., 4(65), 3871-3874.

24- Chelala E, Dirani A, Fadlallah A et al. (2015): Effect of hemodialysis on visual acuity, intraocular pressure, and macular thickness in patients with chronic kidney disease. Clin Ophthalmol., 9: 109-114. 